\title{
Dendritic cells serve as a "Trojan horse" for oncolytic adenovirus delivery in the treatment of mouse prostate cancer
}

\author{
Zhao-lun $\mathrm{LI}^{1, \#}$, Xuan LIANG ${ }^{2, \#}$, He-cheng $\mathrm{LI}^{1}$, Zi-ming WANG ${ }^{1}$, Tie CHONG ${ }^{1, *}$ \\ ${ }^{1}$ Department of Urology, the Second Affiliated Hospital, Xi-an Jiaotong University Medical College, Xi-an 710004, China; ${ }^{2}$ Department of \\ Oncology, the First Affiliated Hospital, Xi-an Jiaotong University Medical College, Xi-an 710061, China
}

\begin{abstract}
Aim: Adenovirus-mediated gene therapy is a novel therapeutic approach for the treatment of cancer, in which replication of the virus itself is the anticancer method. However, the success of this novel therapy is limited due to inefficient delivery of the virus to the target sites. In this study, we used dendritic cells (DCs) as carriers for conditionally replicating adenoviruses (CRAds) in targeting prostate carcinoma (PCa).

Methods: Four types of CRAds, including Ad-PC (without PCa-specific promoter and a recombinant human tumor necrosis factor, rmhTNF, sequence), Ad-PC-rmhTNF (without PCa-specific promoter), Ad-PPC-NCS (without an rmhTNF sequence) and Ad-PPC-rmhTNF, were constructed. The androgen-insensitive mouse PCa RM-1 cells were co-cultured with CRAd-loading DCs, and the viability of RM-1 cells was examined using MTT assay. The in vivo effects of CRAd-loading DCs on PCa were evaluated in RM-1 xenograft mouse model. Results: Two PCa-specific CRAds (Ad-PPC-NCS, Ad-PPC-rmhTNF) exhibited more potent suppression on the viability of RM-1 cells in vitro than the PCa-non-specific CRAds (Ad-PC, Ad-PC-rmhTNF). In PCa-bearing mice, intravenous injection of the PCa-specific CRAd-loading DCs significantly inhibited the growth of xenografted tumors, extended the survival time, and induced T-cell activation. Additionally, the rmhTNF-containing CRAds exhibited greater tumor killing ability than CRAds without rmhTNF.

Conclusion: DCs may be an effective vector for the delivery of CRAds in the treatment of PCa.
\end{abstract}

Keywords: prostate carcinoma; conditionally replicating adenoviruses; PSA; CD4OL; recombinant human tumor necrosis factor; dendritic cells; RM-1 cells; xenograft mouse model

Acta Pharmacologica Sinica (2016) 37: 1121-1128; doi: 10.1038/aps.2016.59; published online 27 Jun 2016

\section{Introduction}

Prostate carcinoma $(\mathrm{PCa})$ is one of the most frequently diagnosed malignant tumors in elderly males ${ }^{[1]}$. Radical prostatectomy and radiation therapy are the commonly used clinical methods for the treatment of PCa. However, up to $40 \%$ of patients who are treated with surgery and radiation may eventually relapse ${ }^{[2]}$, resulting in the migration of cancer cells to other areas of the body. Androgen-deprivation therapy is the preferred treatment for invasive and migratory $\mathrm{PCa}^{[3]}$. While PCa patients initially respond well to androgen deprivation, unfortunately, almost all patients will experience recurrence within 1-3 years due to the development of androgenindependent cancer cells ${ }^{[4]}$. Therefore, there is a clear clinical

\footnotetext{
\#These two authors contributed equally to this work.

* To whom correspondence should be addressed.

E-mail tiechongsx@163.com

Received 2015-11-19 Accepted 2016-02-18
}

need for new methods that can control the recurrence, progression and metastasis of PCa.

Conditionally replicating adenoviruses (CRAds) are now being explored as cancer gene therapy delivery vehicles because they allow viruses to be replicated selectively in predetermined target tumor cells, thereby enhancing the spread of therapeutic gene expression and offering an optimal strategy for cancer treatment ${ }^{[5]}$.

Almost all research into CRAd-mediated PCa gene therapy is limited to local PCa, owing to ease of administration by intratumoral injection and local copying in the prostate, which means that amplification produces fewer side effects. Obviously, intratumoral injection is no longer applicable for advanced PCa with distant metastasis to bone or other organs. Intravenous injection might be one option to solve this problem. However, the intravenous injection of CRAds has produced more difficulties. Because blood is a relatively closed and exclusive system, a series of non-specific and specific host 
defenses removes virus particles from the circulation, producing a major obstacle to achieving effective systemic delivery ${ }^{[6]}$. Dendritic cells (DCs) are professional antigen-presenting cells (APCs) that play critical roles in the activation of antigenspecific naïve $\mathrm{T}$ cells ${ }^{[7]}$ and the regulation of the innate immune response $^{[8]}$. The ability of DCs to deliver CRAds to metastatic melanoma cancer cells in vitro and in vivo has been demonstrated in a previous report ${ }^{[6]}$. CD40 is highly expressed on the surface of DCs. The CD40-CD40 ligand (CD40L) interaction plays an important regulatory role aspects of in DC function such as T-lymphocyte activation and effector cytokine production $^{[9]}$. Researchers have shown that arming an oncolytic virus with CD40L brings the tumor antigens to the DC surface by CD40L, thereby initiating more effective anti-tumor immunity ${ }^{[10]}$.

In the present study, we fused the prostate-specific antigen (PSA) with CD40L and constructed PCa-specific CRAds. Using the DCs as CRAd carriers, the results suggested that CRAd-loading DCs have significant activity against PCa cells and may represent a promising new therapy for PCa.

\section{Materials and methods}

\section{Animals, cells and cell culture}

Male C57BL/ 6 mice (body weight: 16-18 g) at 4-6 weeks of age were obtained from Institute of Materia Medica, Chinese Academy of Medical Sciences \& Peking Union Medical College (Beijing, China). The human transformed embryonic kidney (HEK)-293 cell line and an androgen-insensitive MHC class I-deficient mouse PCa cell line, RM-1, which is syngeneic in C57BL/6 mice ${ }^{[11]}$, were obtained from the American Type Culture Collection (ATCC; Manassas, VA, USA). The cells were cultured in Dulbecco's minimum essential medium (MEM, Mediatech Inc; Manassas, VA, USA), containing 10\% fetal bovine serum (FBS; Gemini Bioproducts; Woodland, CA, USA) and $1 \%$ antibiotic-antimycotic solution (Mediatech Inc; Manassas, VA, USA) $)^{[12]}$ and were maintained at $37^{\circ} \mathrm{C}$ in $5 \%$ $\mathrm{CO}_{2}$ in a fully humidified incubator.

The protocols were approved by the Committee for Ethical Procedures of the Second Affiliated Hospital, Xi-an Jiaotong University Medical College, and the study was performed in accordance with the Declaration of Helsinki (2008) for humans.

Table 1. Primers for overlap PCR.

\section{Construction of Ad-PC, Ad-PC-rmhTNF, Ad-PPC-NCS and Ad-PPC-} rmhTNF

The prostate-specific oncolytic adenovirus was prepared according to a previous report ${ }^{[5]}$. First, we designed and constructed a prostate-specific promoter PSAe-PSMAeTARPp (PPTp), which comprised the prostate-specific antigen enhancer (PSAe), prostate-specific membrane antigen enhancer (PSMAe) and T-cell receptor g-chain alternate reading frame protein promoter (TARPp) in that order. Second, recombinant adenovirus Ad-PSA-CD40L-PPT was prepared using an oncolytic adenovirus preparation system, which was established by our laboratory. The fusion protein gene PSA-CD40L was constructed into a plasmid, and then this sequence was inserted into pShuttle-cmv to obtain pShuttle-PSA-CD40L. The helper plasmid TE-SV-PPT-IR-55K for oncolytic adenovirus preparation was constructed by replacing the TP gene of TE-SV-IR-TP55K with PPTp. Thereafter, the restriction enzyme Mfe I was used to cut TE-SV-PPT-IR-55K and obtain PPT-E1A, and then, the fragment was inserted into the pshuttle-PSA-CD40L in the forward direction to obtain the recombinant plasmid pshuttlePSA-CD40L-PPT-E1A. Finally, the immune and oncolytic adenovirus Ad-PPT-PSA-CD40L (Ad-PPC) was prepared using the Adeasy system (Agilent Technologies, Santa Clara, CA, USA). Ad-PPC was purified by density gradient centrifugation of cesium chloride. The same method was used to obtain the control adenovirus Ad-PSA-CD40L (Ad-PC).

The TNF mutant (recombinant mutated human TNF; rmhTNF) was prepared according to a previous report ${ }^{[13]}$. Briefly, the primers were designed according to TNF-a (GenBank) (Table 1), using the rhTNF-a gene as a template, with amplification of the rmhTNF sequence being achieved by overlap PCR. Double digestion of the IRES from the plasmid pIRES-EGFP was performed, and then mhTNF and a nonsense control sequence (NCS) were connected to IRES. The latter was inserted into the adenovirus Ad-PPC backbone plasmid, and then the resultant plasmid was linearized using the restriction enzyme Pac I before liposomes were transfected into HEK293 cells to obtain the CRAds, Ad-PPC-rmhTNF and Ad-PPC-NCS. The prostate-specific promoter PPT-free control CRAd Ad-PC-rmhTNF was constructed synchronously. All of the recombinant adenoviruses are shown in Figure 1.

\begin{tabular}{|c|c|c|}
\hline 1 & TGG AAT TCA TGT CTC GAA CCC GTA AAC GTA AG & $\begin{array}{l}\text { Containing the initiation codon and ECOR I recognition sequence, replacing the } \\
\text { CCG which encode the eighth amino by CGT, replacing AGT which encode the } \\
\text { ninth amino by AAA, replacing GAT which encode the ninth amino by CGT. }\end{array}$ \\
\hline 2 & ACC CGT AAA CGT AAG CCT GTA GCC CAT GTT & Partly overlap with primer 1 \\
\hline 3 & GTG GTC GAA CGA TCG GGC CAA TGC CCT CC & $\begin{array}{l}\text { Replacing the CTG which encode the twenty-ninth amino by TCG, replacing CGC } \\
\text { which encode the thirty-first amino by GAT. }\end{array}$ \\
\hline 5 & TCA AAA GGC AAT GAT CCC AAA G & $\begin{array}{l}\text { Replacing the TAA which encode the one hundred and fifty seventh amino by } \\
\text { AAA, and containing termination codon and Bam } \mathrm{H} \text { I recognition sequence }\end{array}$ \\
\hline
\end{tabular}




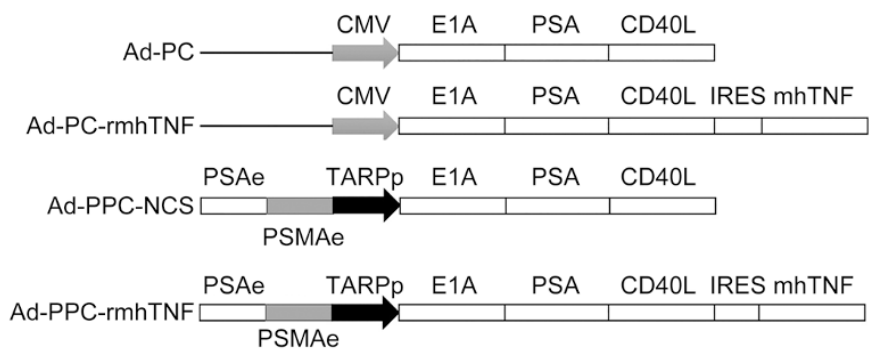

Figure 1. Schematic outline of the recombinant adenoviruses. CMV, immediate-early human cytomegalovirus promoter; E1A, E1A coding sequence; PSA, PSA coding sequence; CD4OL, CD4OL coding sequence; PSAe, PSA enhancer; PSMAe, PSMA enhancer; TARPp, TARP promoter; IRES, IRES coding sequence; mhTNF, human TNF- $\alpha$ coding sequence.

\section{Murine DC-loaded CRAds}

The generation of bone marrow-derived DCs was performed as described previously ${ }^{[14]}$. Femur and tibia marrow cells from C57BL/ 6 mice were depleted of erythrocytes using red blood cell lysis buffer (Sigma-Aldrich, St Louis, MO, USA). The cells were then cultured in complete RPMI-1640 media containing $10 \%$ fetal bovine serum, $10 \mathrm{ng} / \mathrm{mL}$ GM-CSF and $10 \mathrm{ng} / \mathrm{mL}$ IL-4. On d 2, the floating cells were discarded, and the adherent cells were replenished with fresh complete media. On d 4, the culture supernatants were collected and centrifuged, and the cell pellets were resuspended in fresh RPMI-1640 before being returned to the original plate. On d 6, adherent DCs were incubated with B16-F10 cell lysate $(50 \mu \mathrm{g} / \mathrm{mL})$ for $24 \mathrm{~h}$ and then were matured by the addition of $1 \mu \mathrm{g} / \mathrm{mL}$ lipopolysaccharide (Sigma-Aldrich) for $24 \mathrm{~h}$. Mature DCs were harvested and phenotypic markers, such as MHC II, CD80 and CD86 of DCs, were confirmed by fluorescence-activated cell sorting analysis. For the DC-loaded CRAd experiments, $1 \times 10^{6}$ DCs were infected with 0, 5, 10, 50, 100, 200 MOI of CRAd attached with green fluorescent protein (GFP). Ad-PC, AdPC-rmhTNF, Ad-PPC-NCS and Ad-PPC-rmhTNF $(n=10)$ were suspended in $100 \mu \mathrm{L}$ of PBS. After $24 \mathrm{~h}$ of infection, the infection efficiency was detected by fluorescence detection.

\section{Establishment of a mouse PCa model}

Mouse PCa models were generated by the injection of $0.2 \mathrm{~mL}$ of $5 \times 10^{6} / \mathrm{mL}$ RM- 1 cells into the flanks of male C57BL/ 6 mice. After tumors were grown for at least 2 weeks after implantation, 40 mice were randomly divided into 4 different treatment groups (10 mice in each group), and $1 \times 10^{6} \mathrm{DCs}$, which had been infected with $200 \mathrm{MOI}$ of Ad-PC $(n=20)$, Ad-PC-rmhTNF $(n=20)$, Ad-PPC-NCS $(n=20)$ or Ad-PPC-rmhTNF $(n=20)$ suspended in $100 \mu \mathrm{L}$ of PBS, were intravenously injected into the lateral tail vein. Ten other mice were selected as positive controls and were injected with $100 \mu \mathrm{L}$ of PBS 2 weeks after tumor implantation. Fourteen days after CRAd or PBS injection, the tumor volumes of each group were measured to assess the effect of DCs loaded with CRAds on tumor growth. The tumor volume was calculated using the following formula: $v=l \times b^{2} / 2$, where $l$ indicates the long diameter, and $b$ indicates the short diameter. Additionally, survival analysis was performed using the Kaplan-Meier method.

\section{Cell viability and proliferation}

DC viability after isolation was determined using the standard Trypan blue exclusion test (Sigma-Aldrich, St Louis, MO, USA). The total number of viable and nonviable cells was counted using a Bürker counting chamber, and the percentage of viable cells was calculated. The RM-1 viability and peripheral blood mononuclear cell proliferation were measured using the MTT assay. Briefly, the cells were seeded into 96-well plates and washed twice with PBS, and $10 \mu \mathrm{L}$ of MTT was added to each well. Next, the cells were incubated at $37^{\circ} \mathrm{C}$ for $2 \mathrm{~h}$, and $100 \mu \mathrm{L}$ of DMSO was added to dissolve the formazan crystals. The absorbance was measured at $560 \mathrm{~nm}$ using a Spectra Max Paradigm Multi-Mode Reader (Molecular Devices, Austria). The proliferation of peripheral blood mononuclear cells was also studied using the intracellular expression of the nuclear Ki-67 antigen (BD Pharmingen) by flow cytometric analysis. In each assay, $5 \times 10^{5}$ mononuclear cells were incubated with $100 \mu \mathrm{L}$ of cytofix/cytoperm (BD Pharmingen) for 15-20 min on ice. The cells were washed twice with perm/wash buffer (BD Pharmingen) and incubated with an anti-Ki-67 PE antibody (or the matched isotype control) diluted in perm/wash buffer for $30 \mathrm{~min}$ on ice in the dark. Next, the cells were washed again with the perm/wash buffer, resuspended in PBS and analyzed by flow cytometry. The values are expressed as the percentage of stimulated cells positive for the Ki-67 mAb corrected for the percentage of stimulated cells that were positively stained by the isotype control.

\section{Mouse spleen T-cell subset analysis}

Mouse spleen cell suspensions were prepared from spleen samples. The spleen cells were applied to nylon wool columns, and T cells were collected as previously described ${ }^{[15]}$. Mouse spleen $\mathrm{T}$ cell subset analysis was performed by double staining with APC and PE (BD Biosciences, San Diego, CA, USA). Spleen T cells were incubated with APC-conjugated anti-CD4 and PE-conjugated anti-CD8. After an incubation period of $15 \mathrm{~min}$, the cells were analyzed on a flow cytometer (FACSCanto II; BD Biosciences). The percentages of cells expressing CD4 and CD8 were then calculated.

\section{Surface markers}

The surface markers of DCs were detected by flow cytometry. The cells were detached with trypsin-EDTA (Invitrogen) and resuspended in PBS containing 1\% BSA and $0.1 \%$ sodium azide. The samples were incubated for $1 \mathrm{~h}$ on ice with antiCD80, anti-CD86 and anti-MHC II antibodies (Abcam, Cambridge, UK). After washing with PBS, cell staining was carried out for another 1-h incubation with fluorescent anti-rabbit immunoglobulin G (Abcam, Cambridge, UK). The stained cells were analyzed on a FACSCalibur 440E (Becton Dickinson) using Cell Quest software (BD Biosciences Immunocytometry Systems). 


\section{Survival analysis}

Two weeks after RM-1 injection (as described above), the mice were injected intravenously in the lateral tail vein with or without $1 \times 10^{6}$ DCs that had been infected with $200 \mathrm{MOI}$ of Ad-PC, Ad-PC-rmhTNF, Ad-PPC-NCS or Ad-PPC-rmhTNF $(n=10)$ suspended in $100 \mu \mathrm{L}$ of PBS. Mice treated with PBS served as positive controls. All of the mice were followed daily until death.

\section{Statistical analysis}

Data are presented as the mean \pm standard error (SEM) of the data points. Statistical analysis was performed using Student's t-test or one-way ANOVA followed by post-hoc tests and LSD using SPSS 19.0 software. Survival analysis was performed using the Kaplan-Meier method. Data were considered to be statistically significant at a $P$ value less than 0.05 .

\section{Results}

Identification of the load efficiency and safety of CRAds on DCs One prerequisite for exploiting DCs as carriers for CRAds is to identify a CRAds agent that combines a maximum load efficiency with minimum toxicity for DC viability. Therefore, we sought to identify an exact dose of CRAds for DC loading using gradually increasing concentrations of virus. In our experiments, four types of CRAds, including Ad-PC (without a PCa-specific promoter and rmhTNF sequence), Ad-PCrmhTNF (without a PCa-specific promoter), Ad-PPC-NCS (without a rmhTNF sequence) and Ad-PPC-rmhTNF, were constructed as described in the Materials and methods section, and the DCs were infected with various doses $(0,5,10,50,100$ or $200 \mathrm{MOI}$ ) of these viruses. At $24 \mathrm{~h}$ after the infection, the fluorescence intensity in each group was detected under a fluorescence microscope. As shown in Figure 2A, the fluorescence intensity in CRAd-infected DCs increased in a dose-dependent manner despite the different types of viruses. Notably, the percentage of GFP-positive cells accounted for up to a maximum amount of $90 \%$ when the DCs were infected with 200 MOI of CRAds. Additionally, no significant difference in the infection efficiency was found among the four groups of DCs infected with the different types of CRAds. Furthermore, we
A
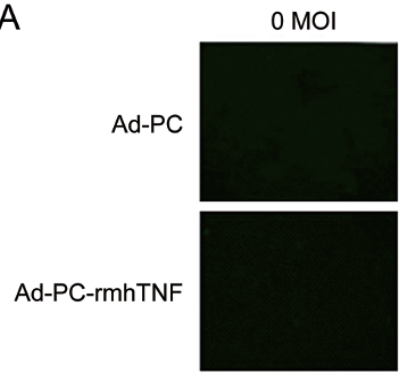

Ad-PPC-NCS

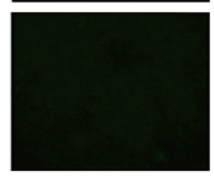

Ad-PPC-rmhTNF



$5 \mathrm{MOI}$
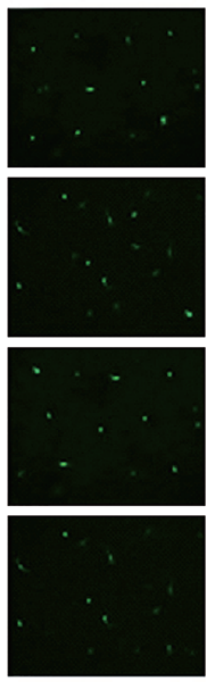

$10 \mathrm{MOI}$
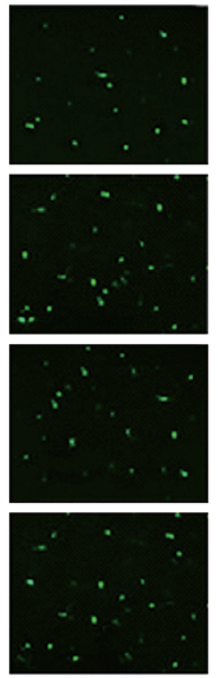

$50 \mathrm{MOI}$
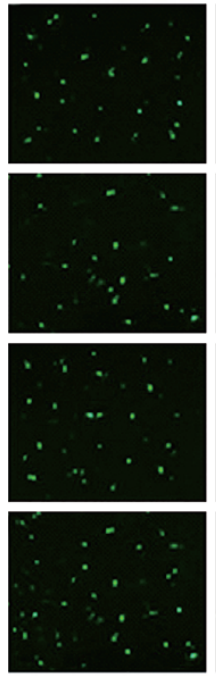

$100 \mathrm{MOI}$
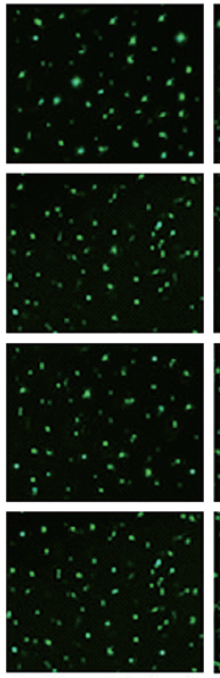

$200 \mathrm{MOI}$
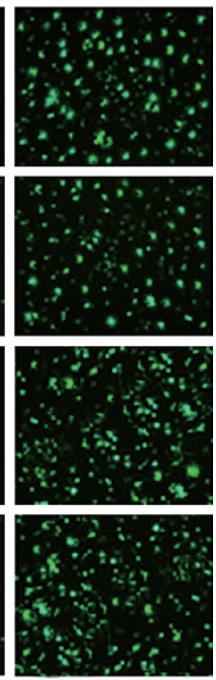

B

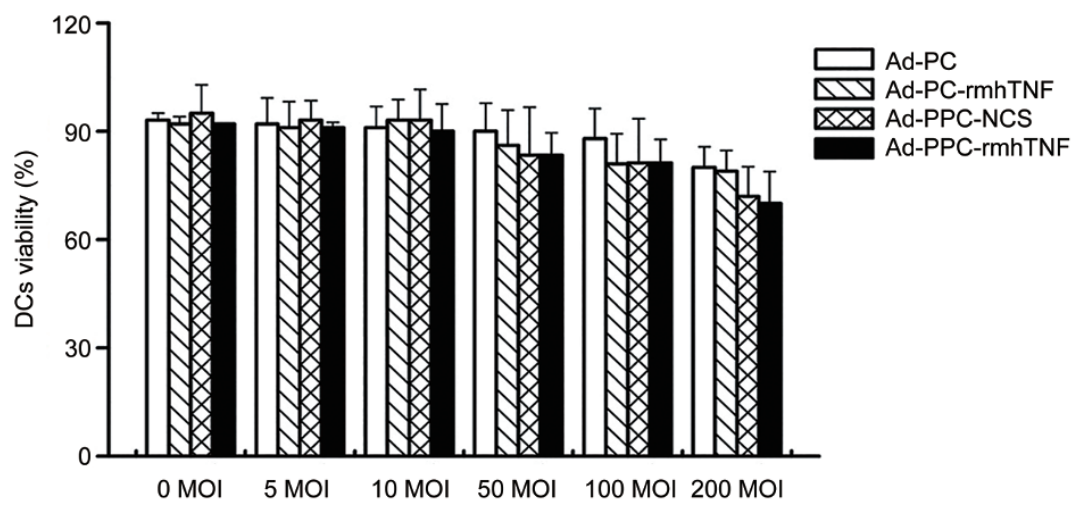

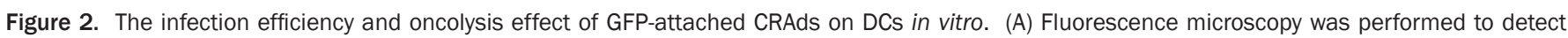

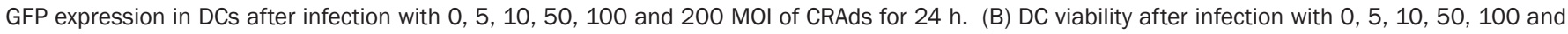
$200 \mathrm{MOI}$ of CRAds for $24 \mathrm{~h}$. 
determined the effects of these four CRAds on the viability of DCs using the MTT assay. The results showed that after infection with 0 to $200 \mathrm{MOI}$ of CRAds, the viability of DCs did not alter significantly (Figure 2B). Therefore, the dose of $200 \mathrm{MOI}$ was appropriate for CRAd infection with limited toxicity on DCs. Based on these data, we selected this dose of CRAd for DC loading in our subsequent experiments.

\section{Effect of CRAd loading on DC maturation}

DCs present antigens to naïve T lymphocytes, contributing to the initiation, suppression or termination of the adaptive immune respons $\mathrm{e}^{[16]}$. DC maturation is critical for antigen presentation and is characterized by the high expression of major histocompatibility complex class II (MHC II) and costimulatory molecules, including CD80 and CD86, etc ${ }^{[17,18]}$. MhTNF-a, a recombinant and mutant $\mathrm{TNF}-\mathrm{a}$, has been reported to accelerate DC maturation with low toxicity to organisms. To compare the effects of CRAds with or without the mhTNF-a sequence on DC maturation, MHC II, CD80 and CD86 expression in CRAd-infected DCs was analyzed. As shown in Figure 3A, mhTNF-a expression was higher in cells infected with AdPC-rmhTNF or Ad-PPC-rmhTNF than in cells infected with Ad-PC or Ad-PPC-NCS. Accordingly, the results showed that the expression of MHC II, CD80 and CD86 was not significantly different between Ad-PC- and Ad-PPC-NCS-loaded DCs (Figure 3B, 3C and 3D). Compared with Ad-PC and AdPPC-NCS infection, infection with Ad-PC-rmhTNF and AdPPC-rmhTNF significantly elevated the expression of MHC II, CD80 and CD86, suggesting that mhTNF-a expression after CRAd infection promotes DC maturation (Figure 3B, 3C and 3D).
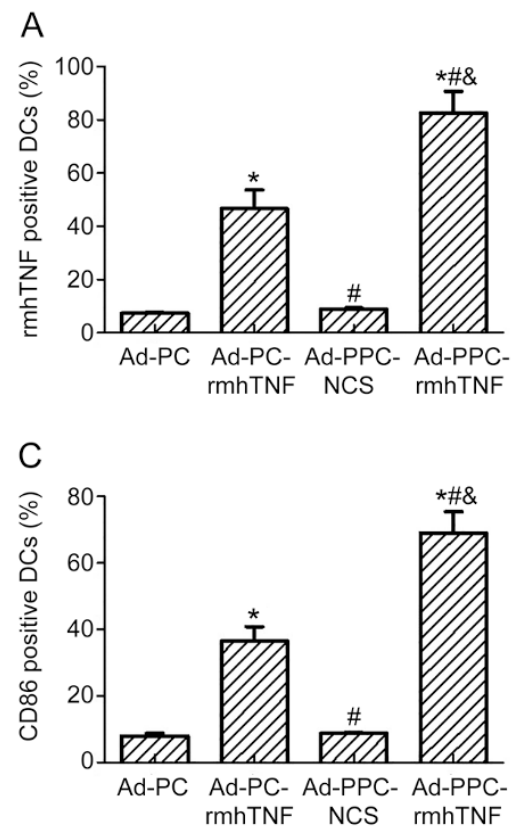

\section{Oncolysis of PCa by CRAd-loaded DCs in vitro}

To investigate the effect of these four groups of CRAd-loaded DCs on the viability of the prostate cancer cell line RM- 1 , we detected the expression of the PSA-CD40L fusion protein and mhTNF- $a$ in RM-1 cells by flow cytometry and determined cell viability after coculturing with CRAd-loading DCs by the MTT assay. As shown in Figure 4A, RM-1 cells co-cultured with non-specific CRAd (Ad-PC or Ad-PC-rmhTNF)-loading DCs exhibited low PSA-CD40L expression. However, after co-culturing with Ad-PPC-NCS- or Ad-PPC-rmhTNF-loading DCs, the percentage of PSA-CD40L+ RM-1 cells increased in a dose-dependent manner (Figure 4A). The percentage of mhTNF+ cells remained unchanged after co-culturing with Ad-PC- or Ad-PPC-NCS-loading DCs but was increased by co-culturing with Ad-PC-rmhTNF-DCs (Figure 4B). Additionally, the results of the MTT assay showed that RM-1 cells were more sensitive to Ad-PPC-NCS- and Ad-PPC-rmhTNF-loading DCs than to those with non-PPT promoter-containing CRAds (Ad-PC and Ad-PC-rmhTNF); the most prominent oncolysis was attributed to Ad-PPC-rmhTNF-loading DCs (Figure 4C). These results demonstrated that Ad-PPC-NCS- and Ad-PPCrmhTNF-loading DCs exhibited the most efficient cytotoxicity on RM-1 cells.

\section{CRAd-loaded DCs influence tumor growth and the survival of tumor-bearing mice}

Tumor volume is an important prognostic factor for $\mathrm{PCa}^{[18]}$. To determine the effect of CRAd-loading DCs on PCa growth in mice, we measured the volume of $\mathrm{PCa}$ in mice that were treated with PBS or Ad-PC-, Ad-PC-rmhTNF-, Ad-PPC-NCS-, or Ad-PPC-rmhTNF-loading DCs. After treatment, the tumor
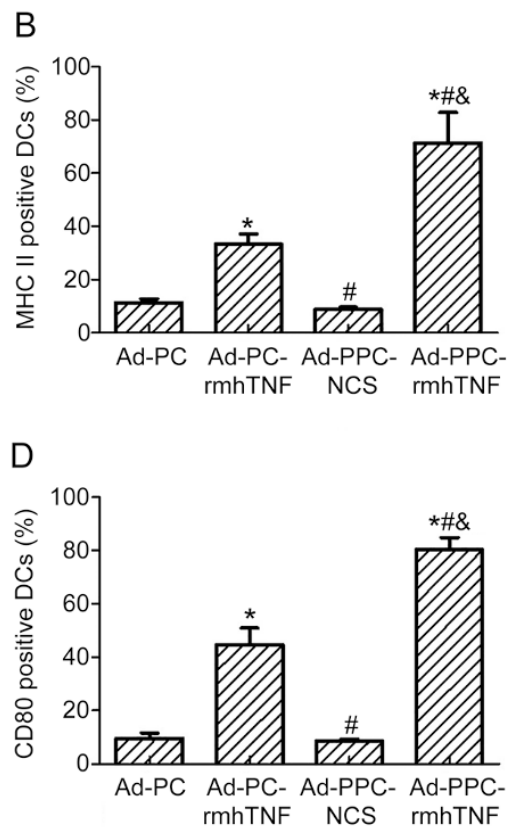

Figure 3. Analysis of surface markers of DCs after infection with $200 \mathrm{MOI}$ of CRAds for $24 \mathrm{~h}$. The percent of rmhTNF- (A), MHC II- (B), CD86- (C) and CD80-positive (D) DCs were detected by fluorescence microscopy. ${ }^{*} P<0.05$ compared with Ad-PC, ${ }^{\#} P<0.05$ compared with Ad-PC-rmhTNF, ${ }^{\&} P<0.05$ compared with Ad-PPC-NCS. 

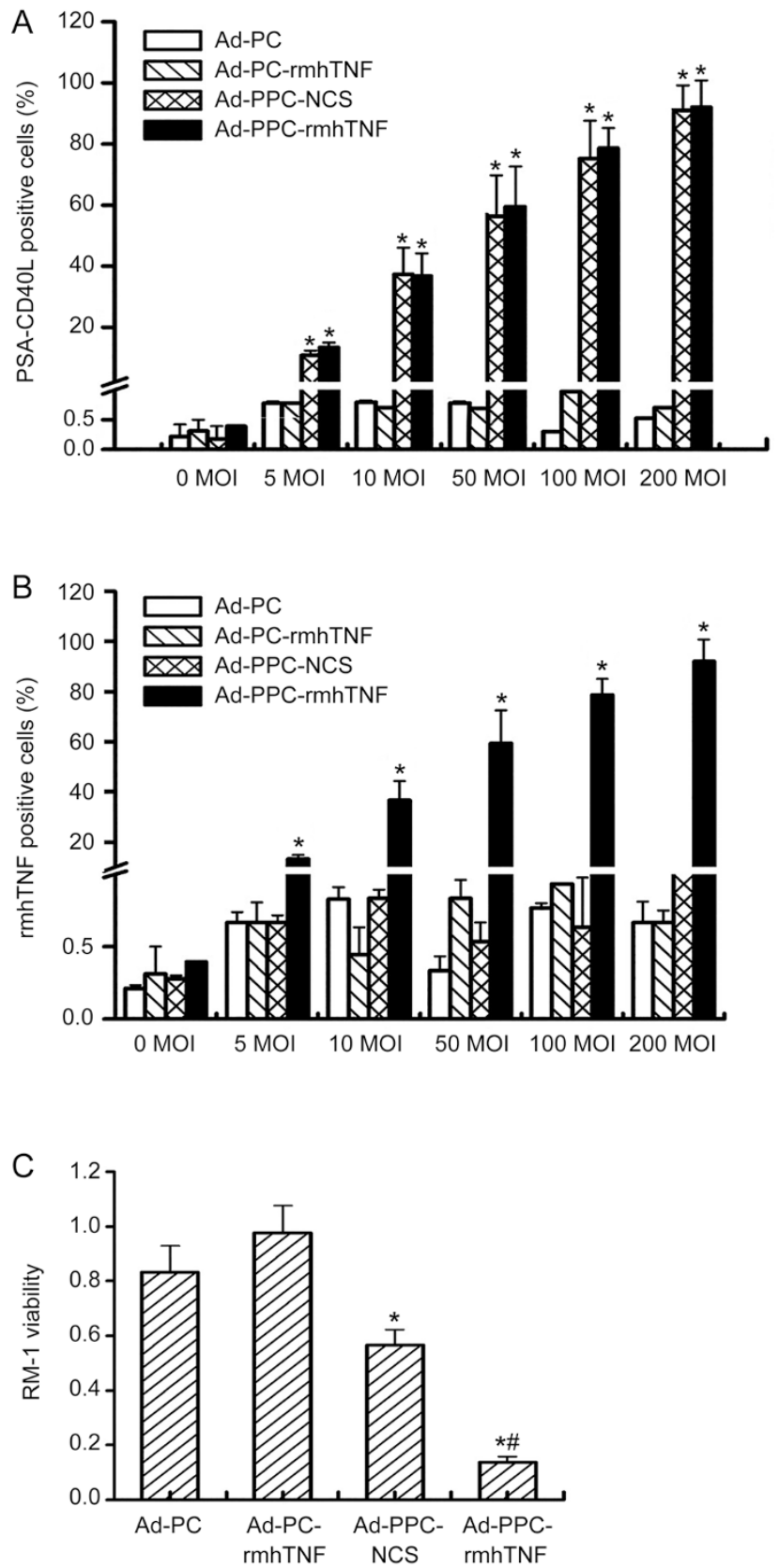

Figure 4. Efficiency and oncolysis effect of four CRAds on RM-1 in vitro. Expression of the fused protein PSA-CD4OL (A) and rmhTNF (B) in RM-1 cells after infection with $0,5,10,50,100$ and $200 \mathrm{MOI}$ of CRAds for $24 \mathrm{~h}$. (C) Oncolysis effect of $200 \mathrm{MOI}$ of different CRAds on RM-1. ${ }^{*} P<0.05$ compared with Ad-PC. ${ }^{\#} P<0.05$ compared with Ad-PPC-NCS.

volumes in the Ad-PPC-NCS and Ad-PPC-rmhTNF groups were significantly smaller $(P<0.05)$ than those in the control, Ad-PC and Ad-PC-rmhTNF groups (Figure 5A). Moreover, treatment with Ad-PPC-rmhTNF-loading DCs resulted in greater tumor growth inhibition than treatment with Ad-PPCNCS-loading DCs (Figure 5A). We next tested whether CRAdloading DCs improved the survival of PCa-bearing mice. As shown in Figure 5B, mice treated with Ad-PPC-NCS- or Ad-
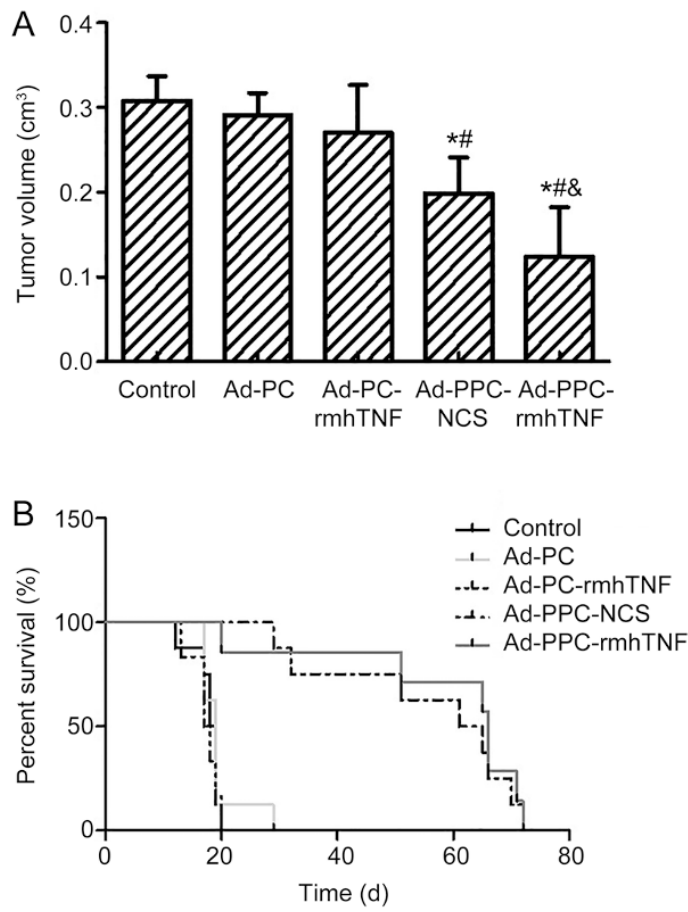

Figure 5. Effect of the intravenous injection of CRAd-loading DCs on prostate tumor volume and mouse survival. (A) Tumor volume after the treatment of mice with CRAd-loading DCs. (B) Mouse survival after the intravenous injection of CRAd-loading DCs. ${ }^{*} P<0.05$ compared with the control. ${ }^{\#} P<0.05$ compared with Ad-PC. ${ }^{\&} P<0.05$ compared with Ad-PPCNCS.

PPC-rmhTNF-loading DCs survived for significantly longer $(P<0.05)$ than those treated with PBS or Ad-PC- or Ad-PCrmhTNF-loading DCs (Figure 5B). Moreover, treatment with Ad-PPC-rmhTNF-loading DCs resulted in a greater survival benefit than treatment with Ad-PPC-NCS-loading DCs (Figure $5 B)$. Thus, these results indicated that the delivery of CRAdloading DCs was efficient for the suppression of tumor growth and improvement of the survival of PCa-bearing mice.

The administration of CRAd-loading DCs activates T cells and reduces $\mathrm{PCa}$ growth

Finally, we examined whether CRAd-loading DCs would activate the immune response of PCa-bearing mice by measuring the differentiation of spleen CD4+ and CD8+ T cells. The results showed that Ad-PPC-NCS- and Ad-PPC-rmhTNFloading DCs could increase the proportion of CD4+ T lymphocytes (Figure $6 \mathrm{~A}$ ) and the $\mathrm{CD} 4+/ \mathrm{CD} 8+$ ratio (Figure $6 \mathrm{~B}$ ) compared with the control and Ad-PC-loading DCs $(P<0.05)$. Moreover, Ad-PPC-rmhTNF-loading DC injection induced a higher $\mathrm{CD} 4+/ \mathrm{CD} 8+$ ratio than Ad-PPC-NCS-loading DC injection (Figure 6A and 6B). Next, we determined the viability of RM-1 cells incubated with spleen T lymphocytes from mice treated with different CRAd-loading DCs. As shown in Figure 6C, T lymphocytes from mice treated with Ad-PPC-NCS- or Ad-PPC-rmhTNF-loading DCs significantly decreased RM-1 cell viability compared with lymphocytes from mice treated 

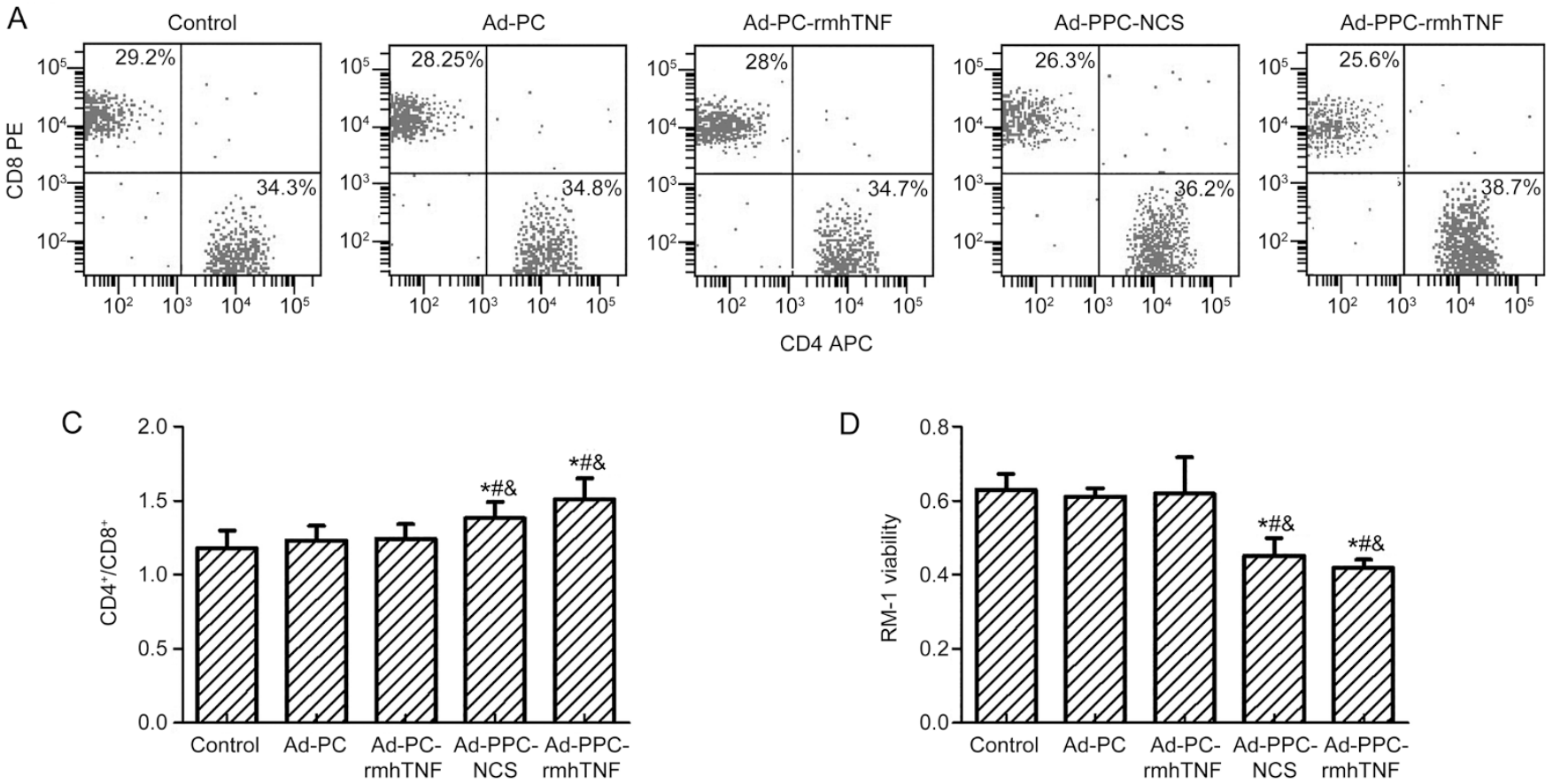

Figure 6. The effect of CRAd-loading DCs on mouse spleen T-lymphocyte subtype and tumor killing ability in vitro. (A) Spleen T-lymphocyte subtype analysis by flow cytometry. (B) $\mathrm{CD}^{+} / \mathrm{CD}^{+}$ratio of spleen $\mathrm{T}$ lymphocytes. (C) RM-1 viability after incubation with spleen $\mathrm{T}$ lymphocytes. ${ }^{*} \mathrm{P}<0.05$ compared with the control. ${ }^{\#} P<0.05$ compared with Ad-PC. ${ }^{\&} P<0.05$ compared with Ad-PC-rmhTNF.

with PBS or Ad-PC- or Ad-PC-rmhTNF-loading DCs.

\section{Discussion}

CRAds are self-replicating, tumor-selective viruses that can directly lyse cancer cells. The efficiency of these agents has been confirmed by evidence from clinical trials ${ }^{[6]}$. CRAd therapy offers an attractive complementary strategy for localized prostate cancer because CRAds can be administered easily by intratumoral injection and local amplification in the prostate produces relatively few side effects ${ }^{[5]}$. However, the intratumoral injection of CRAds is ineffective for metastatic tumors ${ }^{[6]}$. Using cellular carriers to deliver CRAds will be a choice that can overcome a series of host defenses that remove virus particles from the circulation. DCs are professional APCs and play critical roles in the activation of antigen-specific naïve $\mathrm{T}$ cells and in the regulation of the innate immune response $\mathrm{e}^{[8]}$. The ability of DCs to deliver CRAds to metastatic melanoma cancer cells in vitro and in vivo has been investigated in a previous study ${ }^{[6]}$.

Regarding virotherapy, our CRAds were designed to address the biological requirements of PCa. Enhancement of the specificity of CRAds was achieved by constructing a PCa-specific promoter, PPTp, which comprised the prostatespecific antigen enhancer (PSAe), prostate-specific membrane antigen enhancer (PSMAe) and TARPp. The TP gene of the helper plasmid TE-SV-IR-TP-55K was replaced with PPTp. This CRAd has been shown to exhibit superior infectivity and tumor-selective replication in hormone-dependent and hormone-independent prostate cancer ${ }^{[5]}$.
When exploiting DCs as cellular carriers for CRAds, it is important to consider how to limit viral activity in the carrier cells before the DCs reach the prostate cancer cells. In our study, we selected 200 MOI of CRAds because this amount of virus exhibited limited DC cytotoxicity. We demonstrated that DC-based viral delivery had an oncolytic effect on PCa, activated the immune response, and increased the survival of tumor-bearing animals. These results validated the feasibility of using DCs as cellular carriers for CRAds.

TNF- $\alpha$ is a cytokine produced in the tumor microenvironment mainly by macrophages, endothelial cells and tumor cells ${ }^{[19]}$. It is a multifunctional cytokine that exerts cytotoxic, cytostatic and immune-modulatory effects on malignant tumors. However, the use of TNF-a in therapy is limited by its systemic toxicity. Recently, researchers have produced mhTNF-a, which is an efficient, low-toxicity TNF-a allosteric body that has good prospects in tumor treatment ${ }^{[13]}$. In our study, we demonstrated that DCs with mhTNF-a containing CRAds enhanced the inhibition of tumor growth and promoted the survival of tumor-bearing animals. Moreover, the spleen $\mathrm{T}$ lymphocyte $\mathrm{CD} 4^{+} / \mathrm{CD}^{+}$ratio from prostate cancerbearing mice was partially augmented by DCs with mhTNF-a containing CRAds. Therefore, we suspected that mhTNF- $\alpha$ might play a role in immune activation.

In conclusion, this work provides evidence that DCs could serve as a "Trojan horse" for CRAd delivery in anti-cancer therapy; also, CRAd-loading DCs may serve as a new treatment strategy for hormone-dependent and hormone-independent PCa. 


\section{Acknowledgements}

This work was supported by grants from the National Natural Science Foundation of China (№ 81272846) and the Foundation from the Second Affiliated Hospital, Xi-an Jiaotong University Medical College (№ YJ(ZD)201107 and YJ(ZD)201318).

\section{Author contribution}

Zhao-lun LI and Tie CHONG designed the research; Zhao-lun LI and Xuan LIANG performed the research; He-cheng LI contributed to the analytic tools; He-cheng LI and Zi-ming WANG analyzed the data; Zhao-lun LI and Xuan LIANG wrote the paper.

\section{References}

1 Siegel R, Naishadham D, Jemal A. Cancer statistics, 2013. CA Cancer J Clin 2013; 63: 11-30.

2 Djavan B, Moul JW, Zlotta A, Remzi M, Ravery V. PSA progression following radical prostatectomy and radiation therapy: new standards in the new Millennium. Eur Urol 2003; 43: 12-27.

3 Jeet V, Tevz G, Lehman M, Hollier B, Nelson C. Elevated YKL40 is associated with advanced prostate cancer ( $\mathrm{PCa}$ ) and positively regulates invasion and migration of PCa cells. Endocr Relat Cancer 2014; 21: 723-37.

4 Schenk E, Essand M, Bangma CH, Barber C, Behr JP, Briggs S, et al. Clinical adenoviral gene therapy for prostate cancer. Hum Gene Ther 2010; 21: 807-13.

5 Cheng WS, Dzojic H, Nilsson B, Totterman TH, Essand M. An oncolytic conditionally replicating adenovirus for hormone-dependent and hormone-independent prostate cancer. Cancer Gene Ther 2006; 13: 13-20.

6 Ilett EJ, Prestwich RJ, Kottke T, Errington F, Thompson JM, Harrington $\mathrm{KJ}$, et al. Dendritic cells and $\mathrm{T}$ cells deliver oncolytic reovirus for tumour killing despite pre-existing anti-viral immunity. Gene Ther 2009; 16: 689-99.

7 Schmid MA, Harris E. Monocyte recruitment to the dermis and differentiation to dendritic cells increases the targets for dengue virus replication. PLoS Pathog 2014; 10: e1004541.
$8 \mathrm{Xu} \mathrm{H}, \mathrm{Cao} \mathrm{X}$. Dendritic cell vaccines in cancer immunotherapy: from biology to translational medicine. Front Med 2011; 5: 323-32.

9 Moodycliffe AM, Shreedhar V, Ullrich SE, Walterscheid J, Bucana C, Kripke ML, et al. CD40-CD40 ligand interactions in vivo regulate migration of antigen-bearing dendritic cells from the skin to draining lymph nodes. J Exp Med 2000; 191: 2011-20.

10 Pesonen S, Diaconu I, Kangasniemi L, Ranki T, Kanerva A, Pesonen SK, et al. Oncolytic immunotherapy of advanced solid tumors with a CD4OL-expressing replicating adenovirus: assessment of safety and immunologic responses in patients. Cancer Res 2012; 72: 1621-31.

11 Baley PA, Yoshida K, Qian W, Sehgal I, Thompson TC. Progression to androgen insensitivity in a novel in vitro mouse model for prostate cancer. J Steroid Biochem Mol Biol 1995; 52: 403-13.

12 Williams BJ, Bhatia S, Adams LK, Boling S, Carroll JL, Li XL, et al. Dendritic cell based PSMA immunotherapy for prostate cancer using a CD40-targeted adenovirus vector. PLoS One 2012; 7: e46981.

13 Yan Z, Zhao N, Wang Z, Li B, Bao C, Shi J, et al. A mutated human tumor necrosis factor-alpha improves the therapeutic index in vitro and in vivo. Cytotherapy 2006; 8: 415-23.

14 Huang JH, Zhang SN, Choi KJ, Choi IK, Kim JH, Lee MG, et al. Therapeutic and tumor-specific immunity induced by combination of dendritic cells and oncolytic adenovirus expressing IL-12 and 4-1BBL. Mol Ther 2010; 18: 264-74

$15 \mathrm{Wu} \mathrm{H}$, Kumar A, Miao H, Holden-Wiltse J, Mosmann TR, Livingstone $\mathrm{AM}$, et al. Modeling of influenza-specific $\mathrm{CD} 8^{+} \mathrm{T}$ cells during the primary response indicates that the spleen is a major source of effectors. J Immunol 2011; 187: 4474-82.

16 Liu B, Woltman AM, Janssen HL, Boonstra A. Modulation of dendritic cell function by persistent viruses. J Leukoc Biol 2009; 85: 205-14.

17 Kim HP, Lee YS, Park JH, Kim YJ. Transcriptional and epigenetic networks in the development and maturation of dendritic cells. Epigenomics 2013; 5: 195-204.

18 Wolters T, Roobol MJ, van Leeuwen PJ, van den Bergh RC, Hoedemaeker RF, van Leenders GJ, et al. Should pathologists routinely report prostate tumour volume? The prognostic value of tumour volume in prostate cancer. Eur Urol 2010; 57: 821-9.

19 Bemelmans $\mathrm{MH}$, van Tits LJ, Buurman WA. Tumor necrosis factor: function, release and clearance. Crit Rev Immunol 1996; 16: 1-11. 